\title{
Analytical Modelling of Power Efficient Reliable Operation of Data Fusion in Wireless Sensor Network
}

\author{
Jayasri B. S., G. Raghavendra Rao \\ Department of Computer Science, National Institue of Engineering, India
}

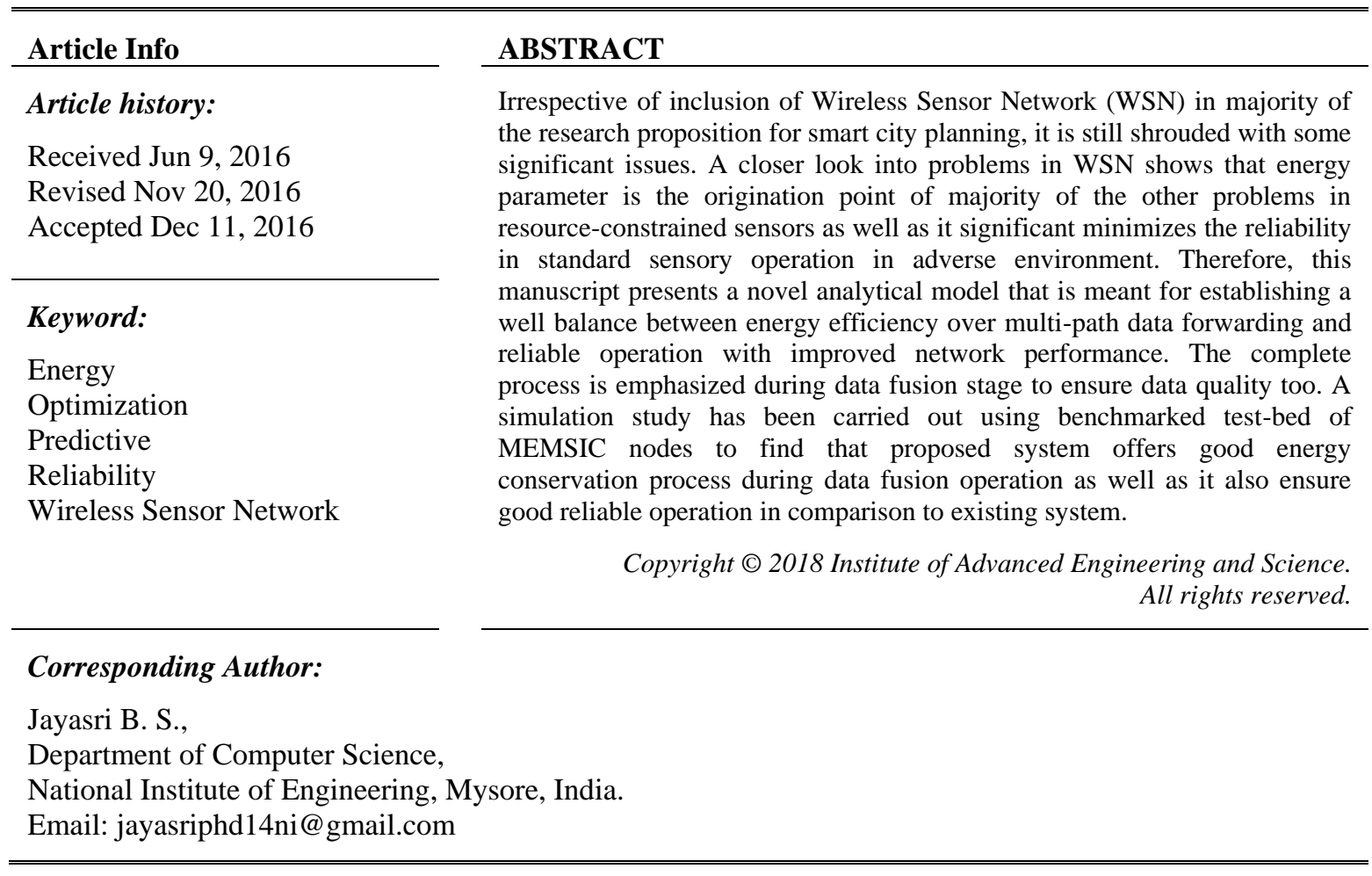

\section{INTRODUCTION}

Wireless Sensor Network (WSN) suits well for performing extraction of physical attributes from the environmental surrounding where it is quite challenging (and sometime impossible) for humans to go [1]. Some of the widely known application of WSN includes monitoring of habitat, tactical information acquisition in military, healthcare-based application, industrial application, etc. [2]. Although, there are reported usages of sensory application since last decades, but there are lots of difference between commercially available products and what is discussed in research-papers. The fact is only 10-30\% of the features discussed in research-based papers actually make is way to the commercial application owing to various form of non-disclosed challenges associated with sensory operation. There are various literatures to claim that WSN has significant problems in routing [3], energy [4], security [5], and traffic-related management [6].

Irrespective of widely available manuscript on research archives, majority of them are more hypothetical and less practical. It was also observed that energy is the root cause of majority of the operational challenges within a sensor node [7]. Basically, a wireless sensor node possesses very limited power in terms of battery as well as they are also resource-constrained. For this reason, if any operation of sensing or data packet forwarding/receiving has to happen, there is a significant consumption of power. Although, power consumption happens at all the stage of data forwarding in WSN, but particularly it is essential to be studied during data fusion and data aggregation process. It is required to be understood that data fusion highly differs from data aggregation. It was also noticed that studies towards data fusion [8] stage in WSN is relatively less in literature as compared to that of data aggregation [9]. Basically, data fusion is a stage that connects all the communicating nodes only to check for unique accumulation of data packet from 
the member node in local level of clustering. However, while doing this task, it is essential to understand the type of applications. If the application inclusion monitoring of uncertain event with no pre-defined time instance (e.g. habitat monitoring, healthcare, etc), in such condition, there may be a situation that the node was idle for a long time with no significant capturing of any potential environmental information. However, problem arise when a specific event has occurred during the time when the nodes have very less residual energy, not good enough to process the complete data packet to the destination node. Hence, this condition states that a node will need to be energy-efficient as well as it is required to ensure higher degree of reliable operation with an extended network life time.

In reality, there is no such research work to jointly address this problem. Without fixing this combined problem, a sensor node will still be vulnerable to other set of problems e.g. routing problems, security problems, encountering bottleneck problems, etc. Therefore, the contribution of the proposed study is to present a novel solution which can offer solution towards the above mentioned problems associated with energy as well as reliable operation of a resource-constraint sensor node in WSN. The presented study has introduced the use of a predictive-based optimization process that uses an analytical modeling in order to construct a novelframework. It also defines exclusive research problems in the consecutive Section 1.2 The fundamental concept about proposed research methodologies to solve the reported research problem is discusses in Section-1.3 that is further followed by system design in Section-2. Section-3 discusses about accomplished outcome whereas Section 4 briefs about final conclusion on the proposed study.

\subsection{Background}

This section adds more information to the existing research approaches towards energy-efficiency in WSN from our prior review work [10]. Based on the extensive review, Jayasri et al. [11] observed that very less studies have been carried out on reliable data dusion and further come up with a novel analytical solution namely framework for reliable data fusion (FRFD) in order to ensure energy-aware data forwading in WSN. The outcome of the study found to excel superior communication performance in terms of energy efficiency and reliable data transmission.

Study towards energy problems as well as data delivery issues have been addressed in the work of Wang et al. [12] with an aid of grid routing mechanism. Study towards energy efficiency problems has been also discussed by Nadas et al. [13] using synchronization based approach in WSN that minimize the window of idle listening. Most recently study towards energy and reliability problems has been addressed by Alshaheen and Takruri-Rizk [14] using network coding approach. Dobslaw et al. [15] have presented a scheduling approach using heuristic-based methodology for ensuring beter reliability in communication of WSN. The work carried out by Fernandes et al. [16] have presented a scheme to minimize transmission power consumption using received signal strength for addressing overhead problems. Oliveira et al. [17] have presented a simplified mechanism for energy saving considering a specific application. The problems of node reliability are also addressed by Zhu et al. [18] in the context of physical layer security.

Zonouz et al. [19] have presented a reliability modeling for single hop communication in WSN using cost-function based approach. Reliability issues were also investigated with respect to fog-computing in order to better establish relationship with sensor devices and cloud environment. Such work has been presented by Wang et al. [20] that perform computation of trust in order to prove reliability of the sensor. Sarma et al. [21] have presented a routing-based approach for addressing energy and reliability problems associated with the communication considering inclusion of mobility factor in WSN. Qadori et al. [22] have used fuzzy-approach along with consideration of mobile agents in order to conserve energy consumption. Usage of context-based sensing mechanism was also proven to affect energy saving as reported in the work of Prabha et al. [23]. Han et al. [24] have investigated coverage problems and its relationship with the energy attribute in WSN. An empirical-modelling based approach was presented by Feng and Dong [25] in order to investigate reliability issue. Elsafrawey et al. [26] have presented an involuntary healing proposition towards ensuring reliability of data in WSN. An approach was presented by Campobello et al. [27] that has utilized a unique remainder theorem for enhancing energy saving features as well as reliability problems among sensory applications.

The work carried out by Dong et al. [28] have discussed about an optimization approach for leveraging the reliability aspect of the WSN. The authors have developed the scheme using a unique data acquisition approach towards addressing better clustering performance with less delay. Usage of network coding for enhancing the energy saving charecteristics in sensor is presented by Liu et al. [29]. The authors have used coding scheme for both network and channel in order to increase the reliability score of the WSN with more signal quality. Study towards data gathering was investigated by Long et al. [30] where a comprehensive mathematical modeling has been introduced in order to ensure the reliability scope of a sensor node working in an adverse condition of networking. The study also claims of optimized outcome 
with respect to energy saving and proper utilization of network. Probems associated with the reliability was also witnessed to be solved using filtering-based technique.

Studies in such direction was carried out by the Pak et al. [31] considering inclusion of finite impulse filter that is constructed for identifying the ode failures in WSN. The authors have used a hybridized design of a filter in order to ensurebetter localization scheme of defectidentification as a medium to assure better reliability scheme in WSN. Therefore, it can be seen that there are various reported scheme of enhancing the reliability score of the WSN using different methodologies. The next section discusses about the identification of the potential problems associated with the existing approaches in next section.

\subsection{The Problem}

The significant research problems are as follows:-

a. Existing approaches towards improving energy efficiency lacks critical optimization process considering computational and network-based resources.

b. Studies towards reliability-based methods are not found to ensure any form of predictive-based analysis without using any form of iterative-based conventional methods.

c. Lack of benchmarked studies towards solving energy as well as reliability problems is another significant research gap in WSN.

d. A comprehensive modeling considering all the practical constraints of a sensor node to assess the node as well as network performance is also missing in existing system.

Therefore, the problem statement of the proposed study can be stated as "To design a comprehensive framework that is capable of offering significant reliable node operation with superior network lifetime in presence of dynamic network scenarios". The next section briefs about solution to address this issue.

\subsection{The Proposed Solution}

The prime purpose of the proposed system is to present a joint framework which enables a simulation environment to assess three different types of modules where firstly, Module-1 represents an analytical methodology well capable of ensuring energy-aware data fusion to address the power consumption issues in a data-driven WSN also abbreviated as EEDF. Secondly, the framework also integrates Module-2 where the prime concern laid upon designing cost-effective computational modelling which ensures power efficient, reliable operations during data fusion process on a large scale WSN using stratistical spreading mechanism (RaESS). Finally, it further optimizes the communication performance during sensor network operations in terms of significant energy efficiency and reliability scores by incorporating predictive evolutionary modelling which is presented in Module-3. The following Figure 1 exhibits the joint framework intended to assess three different modules within a simulation environment.

\section{A Novel Joint Framework for Reliable and Power Efficient Data Fusion Operation in WSN}

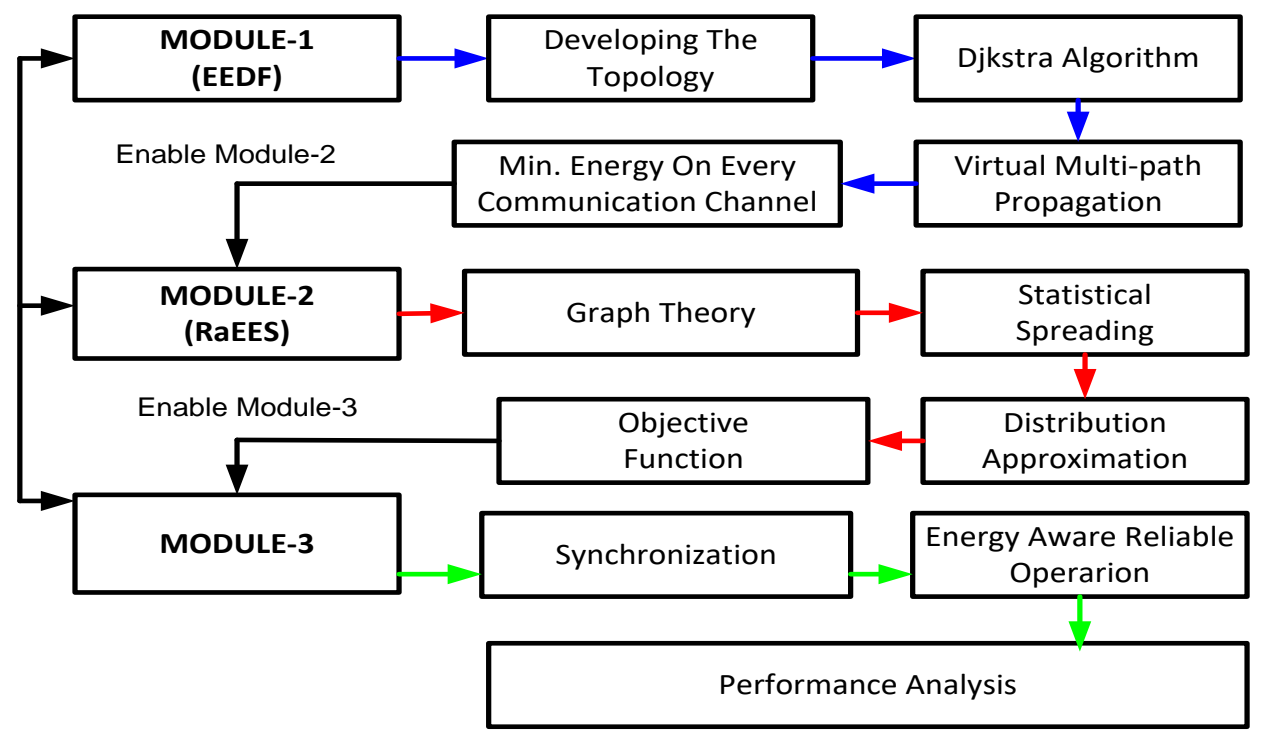

Figure 1. Implemented schema of proposed system 
The above Figure 1 shows the design principles of the proposed analytical methodology to simulate the performance of the joint computational framework. The simulation environment is created using a numerical computing envirpnment which enables evaluating three different modules with respect to their computational flow of design. Finally, the outcome assessed for different modules are compared with the conventional baselines in order to justify the implemented methodology of the proposed system towards accomplishing energy aware reliable operations in a large scale WSN. The more elaborated discussion of the proposed three different modules are presented in the next segment of the manuscript i.e. system design.

\section{SYSTEM DESIGN}

This section concern about discussing the system design methodology of the proposed framework to ensure adequate outcome in the form of numerical solutions.However, it basically aims towards justifying the fact that the study accomplishes efficient and optimal communication operations regarding reliability and energy performance during data fusion operation in a multicast WSN routing. The proposed framework evolved up with a multi-fold methodology which includes basically three different types of modules operating jointly in a numerical computing environment. The following are the extensive elaboration of three separate modules which can carry out reliable operations involved in data fusion and clustering process to utilize optimal energy in a WSN.

a. Design Analysis of Module-1(EEDF): This analytical design methodology for module-1 has been conceptualized to enhance the data fusion process in a WSN. EEDF forumalted keeping a condition into consideration i.e. it should support data fusion process in a multipath propagation system. It basically adopted the concept of antenna theory which ensures higher degree of reliable communication operations by deploying enhanced multipath propagation mechanism. Incorporation of antenna theory in this regards increses the capacity of radio links towards enahcning the supportability of multipath propagation with higher degree of reliable communication operation. In this intial phase the proposed system deployed a WSN topology by evolving up a distributed tree construction supported by graph theory. The graph is constructed using a set of vertices and edges which is expressed with the following mathematical Equation 1. In that eq. $\mathrm{V}$ refers to the set of vertices used to denote sensor nodes and $\mathrm{E}$ belongs to the set of edges which are basiclally communication links within a multipath propagation system. The addition of base station $b$ makes the graph expressed like following:

$$
\mathrm{G}=(V \mathrm{U} b, E)
$$

Where every sensor $n € V$ will have the freedom to choose its adjacent neighbours for mutli-cast routing during ad-hoc communication. The novelty of the proposed concept is that it enables deployment of assistant pair of nodes which supports single and multi-path propagation during the communication between other nodes, this paradigm resulted energy efficient link formation with the supportability of multipath propagation between node and base station. It also optimizes the data transmitted from one sensor node $\mathrm{n}$ to a base station $\mathrm{b}$ by formulating the folloing objective function which helps in energy optimization.

$$
O_{\text {func }}=\arg _{\min } \sum_{n \in V} E_{n}
$$

The experimental observations further assured that this approach has significantly reduced energy requirements during data fusion operations and also potentially assisted in conserving energy to a significant level. The optimum selection of communication channel also assisted in minimizing enery consumpsion during multipath propagation in a virtual topology where on the other hand the data dissemination process reduced the redundancy of data to ensure that unique data being forwarded to the $b$.

b. Design Analysis of Module-2(RaEES): The analytical modellling of RaEES designed and developed by addressing communication performance isssues of a WSN from energy and reliability view point. The design principle of RaEES also implements a numerical analysis which enables reliable data transmission during data fusion process in a data driven WSN. The system aims to ensure an optimal equilibrium between the uninterrupted data transmission prior performing fusion with minimal usage of network and computational rsources. The adopted graph theory modelling used in the formulation of fusion structure with a tree based topology formation which enables reliable communication operations with less resource consumpsion. However, minimal usage of resources during network operations and data aggregation process including elimination of repetitive redundant data significantly improved communication performance in terms of energy and reliability which are further justified in the next

Int J Elec \& Comp Eng, Vol. 8, No. 6, December 2018 : 4637 - 4645 
section 3 with an extensive discussion on experimental analysis. The cocept of statistical spreading has been used to ensure transmission reliability in terms of reducing packet error rate and dropping probability using a distributed approximation paradigm. However, finally the proposed system formulates an objective function which ensures higher degree of reliability while consuming minimal computational and network resources. The next section presents the model analysis of the next methodology from analytical and theoretical perspective [32].

c. Design Analysis of Module-3: This phase of the design methodology exclusively carried out by emplying a decision oriented data delivery model with predictive coding. It basically enhances the communication operations by incorporating a time-slot synchronization modelling where the allotment of time-slots during synchronization phase follows two different mode of operations which are thereby i) busy mode and ii) idle mode of operations respectively. It further applies two different algorithms in order to obtain power efficienct comprehencive paradigm for reducing resource consumpsions during communication operations by which it has certainly achieved better reliability during clustering and data fusion process. It mostly puts emphasize towards classifying the data packets with the deployment of cost-effective Time Division Multiple Access (TDMA) and Carrier Sense Multiple Access (CSMA) subjected to decisional and efficient energy modelling. The proposed system perform data fusion prior to data aggregation when aggregator nodes act as cluster head $\left(C_{h}\right)$ while operating on diffent sensor node (SN) driven clusters. The process thereby mainly follows two different types of communication phases such as i) communication between node to $\mathrm{C}_{\mathrm{h}}$ where data fusion take place and ii) communication between each $\mathrm{C}_{\mathrm{h}}$ to core aggregator mote, data aggregation is performed. The prime emphasize has been given towards energy consumpsion optimization by performing reliable sensory operations with synchronized packet delivery paradigm with randomized and organized access mode of processes. The data delivery cycle has been conceptualized in a way where a time slot is defined for transmission operations, also referred as temporal interval $\left(t_{\mathrm{i}}\right)$. The next segment of the study thereby discussed the outcome being accomplished by simulating the joint framework in a numerical compiuting environment.

\section{RESULT ANALYSIS}

\subsection{Outcomes Obtained from Module-1}

This section demonstrates the experimental outcomes obtained after simulating the proposed joint framework with a numerical analysis. The framework initially evaluates the analytical methodology derived for EEDF and obtains the results pertaining to energy consumpsion per request (mJ) which is highlighted in the following Figure 2. The above Figure 2 clearly exhibits that the module-1 EEDF accomplishes better outcomes in terms of energy with the increasing value of nodes. The proposed EEDF thereby significantly minimizes the energy consumpsion (0-0.3) $\mathrm{mJ}$ in comparison with the conventional baselines LEACH and PEGASIS.

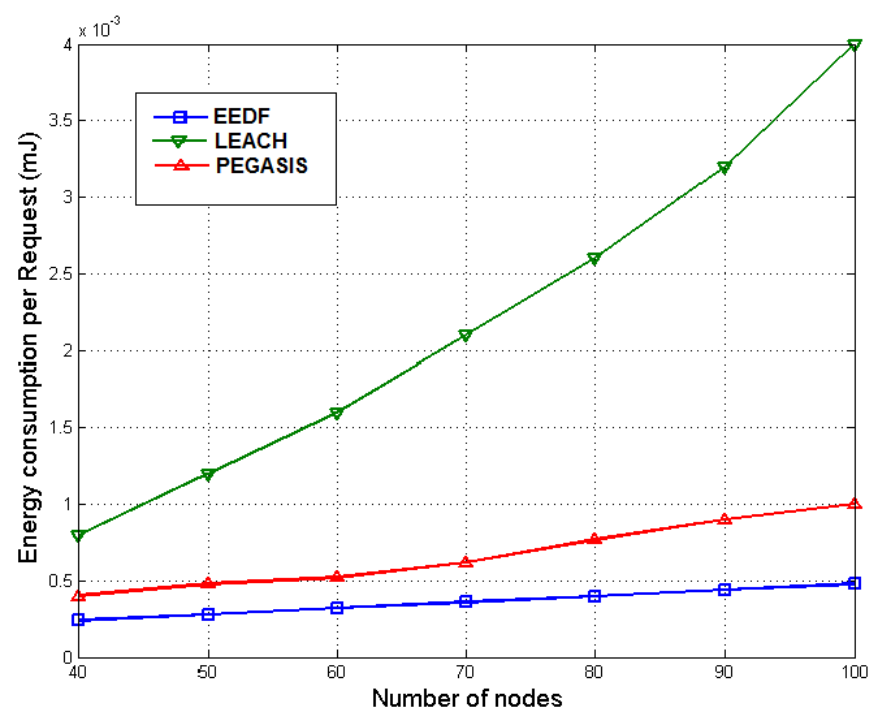

Figure 2. Analysis of energy consumpsion request $(\mathrm{mJ})$ 


\subsection{Outcomes obtained from Module-2}

The evaluation of the study was carried out with 600 sensor nodes compliant with MEMSIC node configuration dispersed randomly under simulation area of $1000 \times 1000 \mathrm{~m}^{2}$. A hypothetical data of 3500 bits has been used for communication purpose. As the proposed RaESS focuses towards achieving reliable transmission, it was essential for evaluating its effectiveness with respect to energy consumption, delay, packet delivery ratio, as well as algorithm processing time.

The following Figure 3, 4 exhibits that how the proposed RaESS obtains better outcomes in terms of packet delivery ratio and overall delay (S) during data fusion process in a WSN communication operations. It also shows that overall delay significantly reduced in RaESS as compared to LEACH.

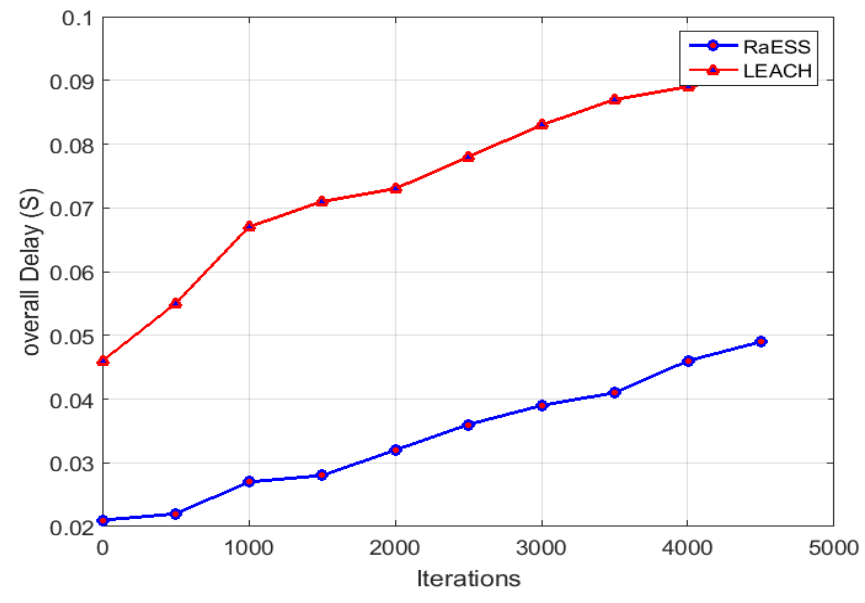

Figure 3. Overall delay vs iterations

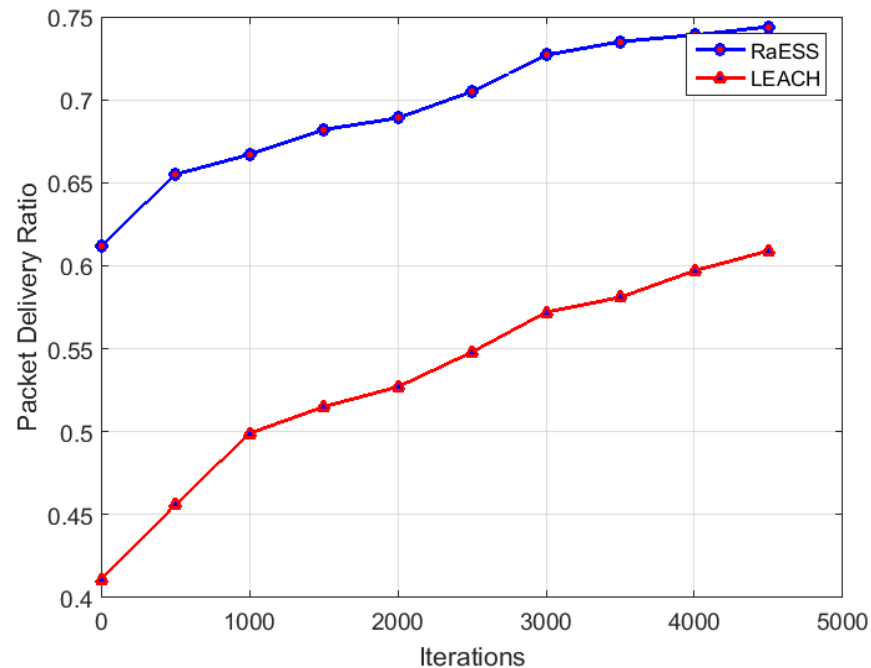

Figure 4. Packet delivery ratio vs iterations

It can also be seen that the proposed RaESS significantly reduces delay to enahce the reloiable network opetrations prior to data fusion while nodes are communicating with their core aggregator mote. The above Figure 4 also shows the efficiency of RaESS in obtaining enhanced packet delivery ratio which also ensures better communication performance during the data fusion phase with optimized outcome in energy factors.

Figure 5 also exhibits that the proposed RaESS attain very lesser computation time as the proposed algorithm consumes very less number of resources during the soft computing phase. The outcome obtained found highly superior than the conventional LEACH algorithm. 


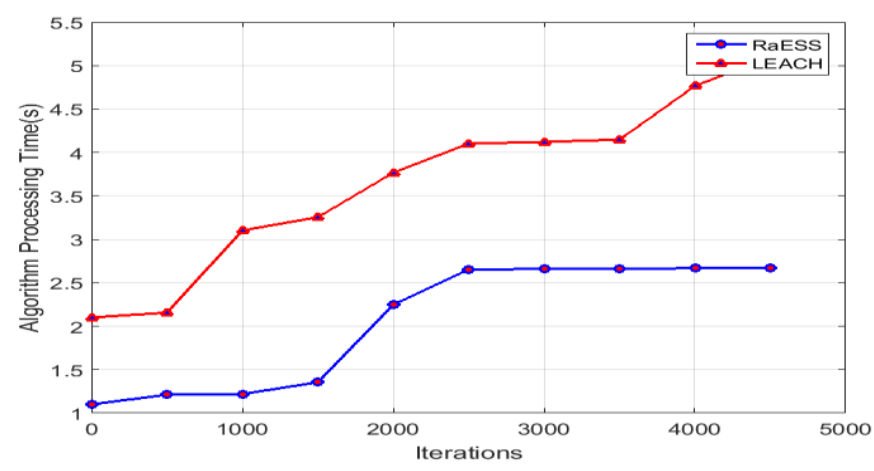

Figure 5. Analysis of algorithm processing time(s)

\subsection{Outcomes obtained from Module-3}

The following Figure 6 also exhibits that the proposed two algorithms for moule-3 also achieves reliable communication operations to reduce the delay constraints which has a significant impact on energy consumpsion optimization. The above Figure 6 also shows that energy consumpsion per bit in every instance of increasing time slots is higher in the case of Luo [33] where, Proposed approach and Zhang [34] approach accomplishes optimal outcome as they both utilized predictive node scheduling in a dynamic environment where nodes are actually performs decision making during scheduling and data fusion process. However, as our proposed system excels better outcomes with respect to different attributes thus the study ensures its uniformity towards achiving reliable communication scenario during data fusion process in a WSN.

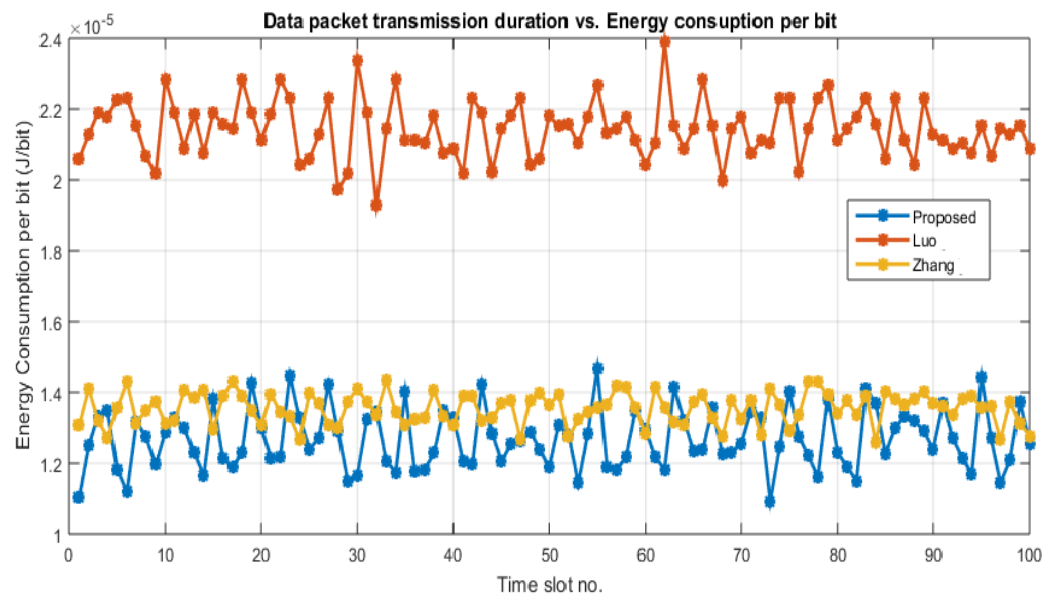

Figure 6. Analysis of energy consumpsion per bit (j/bit)

\section{CONCLUSION}

The current advancement of sensor network operations manly data fusion highly requires reliable computation with minimal usage of resources to ensure enahced communication performance in terms of energy consumpsion and delay. However, sensor network operations in a region of interest is highly unpredictive owing to its decentralized communication pattern, therefore placement of nodes should be within a monitored area in order to track down every respective communication activity. The investigational analysis in our prior works found that very less existing works have been carried out towards ensuring energy efficient data fusion operations in a large scale WSN where no significant focus inclined towards optimizing the reliability factors with respect to delay and energy constraints.

The study formulated a novel joint schema which assess three different analytical methodology to forward data with maximum reliability and minimum usage of energy in a multipath propagation system. It is also ensured that the proposed system utilizes very lesser network resources while carrying out the data fusion supported by energy aware channel access. The study outcome further ensures that the proposed joint framework accomplishes better outocmes in terms of different performance metrices. 


\section{REFERENCES}

[1] \Nouha Sghaier, Abdelhamid Mellouk, Energy Efficient Design of Wireless Sensor Networks, ISTE Press Limited, Elsevier-2018

[2] Dac-Nhuong Le, Raghvenda Kumar, Jyotir Moy Chetterjee, Introductory Concepts of Wireless Sensor Network. Theory and Applications, GRIN Verilag, 2018

[3] X. Liu, "Routing Protocols Based on Ant Colony Optimization in Wireless Sensor Networks: A Survey," in IEEE Access, vol. 5, pp. 26303-26317, 2017.

[4] A. Solayappan, M. B. H. Frej and S. N. Rajan, "Energy efficient routing protocols and efficient bandwidth techniques in Underwater Wireless Sensor Networks - a survey," 2017 IEEE Long Island Systems, Applications and Technology Conference (LISAT), Farmingdale, NY, 2017, pp. 1-7.

[5] B. Mostefa and G. Abdelkader, "A survey of wireless sensor network security in the context of Internet of Things," 2017 4th International Conference on Information and Communication Technologies for Disaster Management (ICT-DM), Münster, 2017, pp. 1-8.

[6] H. Yuan, N. Yugang and G. Fenghao, "Congestion control for wireless sensor networks: A survey," The 26th Chinese Control and Decision Conference (2014 CCDC), Changsha, 2014, pp. 4853-4858.

[7] V. Musale and D. Chaudhari, "Challenges, protocols and case studies in design of reliable energy efficient wireless sensor networks," 2017 4th International Conference on Advanced Computing and Communication Systems (ICACCS), Coimbatore, 2017, pp. 1-7.

[8] C. M. d. Farias and L. Pirmez, "A Multisensor Data Fusion Technique for Multiapplication Wireless Sensor Networks Based on Overlapping Intervals," 2017 IEEE 15th Intl Conf on Dependable, Autonomic and Secure Computing, 15th Intl Conf on Pervasive Intelligence and Computing, 3rd Intl Conf on Big Data Intelligence and Computing and Cyber Science and Technology Congress(DASC/PiCom/DataCom/CyberSciTech), Orlando, FL, 2017, pp. 804-811.

[9] H. Lin, R. Xie and L. Wei, "Density, distance and energy based clustering algorithm for data aggregation in wireless sensor networks," 2017 IEEE/CIC International Conference on Communications in China (ICCC), Qingdao, 2017, pp. 1-5.

[10] Jayashri B. S and G. R. Rao, "Reviewing the research paradigm of techniques used in data fusion in WSN," 2015 International Conference on Computing and Communications Technologies (ICCCT), Chennai, 2015, pp. 83-88.

[11] B.S. Jayasri, G. Raghavendra Rao, "FRDF: Framework for Reliable Data Fusion to Leverage Communication Performance in Sensor Network", Software Engineering Trends and Techniques in Intelligent Systems, pp. 11-22, 2017

[12] K. Wang, H. Gao, X. Xu, J. Jiang and D. Yue, "An Energy-Efficient Reliable Data Transmission Scheme for Complex Environmental Monitoring in Underwater Acoustic Sensor Networks," in IEEE Sensors Journal, vol. 16, no. 11, pp. 4051-4062, June1, 2016.

[13] J. P. B. Nadas, R. D. Souza, M. E. Pellenz, G. Brante and S. M. Braga, "Energy Efficient Beacon Based Synchronization for Alarm Driven Wireless Sensor Networks," in IEEE Signal Processing Letters, vol. 23, no. 3, pp. 336-340, March 2016

[14] H. Alshaheen and H. Takruri-Rizk, "Energy Saving and Reliability for Wireless Body Sensor Networks (WBSN)," in IEEE Access, vol. 6, pp. 16678-16695, 2018

[15] F. Dobslaw, T. Zhang and M. Gidlund, "End-to-End Reliability-Aware Scheduling for Wireless Sensor Networks," in IEEE Transactions on Industrial Informatics, vol. 12, no. 2, pp. 758-767, April 2016.

[16] D. Fernandes, A. G. Ferreira, R. Abrishambaf, J. Mendes and J. Cabral, "A Low Traffic Overhead Transmission Power Control for Wireless Body Area Networks," in IEEE Sensors Journal, vol. 18, no. 3, pp. 1301-1313, Feb.1, 1 2018

[17] F. Denis Mendonca Oliveira, R. Soares Semente, J. Doolan Fernandes, T. Augusto Correia Melo and A. Ortiz Salazar, "SEREE: An Energy-Efficient Wireless Sensor Network Embedded System to be Applied on Plunger Lift Oil Elevation Method," in IEEE Latin America Transactions, vol. 13, no. 4, pp. 1187-1197, April 2015.

[18] J. Zhu, Y. Zou and B. Zheng, "Physical-Layer Security and Reliability Challenges for Industrial Wireless Sensor Networks," in IEEE Access, vol. 5, pp. 5313-5320, 2017

[19] A. E. Zonouz, L. Xing, V. M. Vokkarane and Y. L. Sun, "Reliability-Oriented Single-Path Routing Protocols in Wireless Sensor Networks," in IEEE Sensors Journal, vol. 14, no. 11, pp. 4059-4068, Nov. 2014.

[20] T. Wang et al., "Fog-Based Evaluation Approach for Trustworthy Communication in Sensor-Cloud System," in IEEE Communications Letters, vol. 21, no. 11, pp. 2532-2535, Nov. 2017

[21] H. K. Deva Sarma, R. Mall and A. Kar, "E2R2: Energy-Efficient and Reliable Routing for Mobile Wireless Sensor Networks," in IEEE Systems Journal, vol. 10, no. 2, pp. 604-616, June 2016.

[22] H. Q. Qadori, Z. A. Zukarnain, Z. M. Hanapi and S. Subramaniam, "FuMAM: Fuzzy-Based Mobile Agent Migration Approach for Data Gathering in Wireless Sensor Networks," in IEEE Access, vol. 6, pp. 15643-15652, 2018

[23] R. Prabha, M. V. Ramesh, V. P. Rangan, P. V. Ushakumari and T. Hemalatha, "Energy Efficient Data Acquisition Techniques Using Context Aware Sensing for Landslide Monitoring Systems," in IEEE Sensors Journal, vol. 17, no. 18, pp. 6006-6018, Sept.15, 152017.

[24] G. Han, L. Liu, J. Jiang, L. Shu and G. Hancke, "Analysis of Energy-Efficient Connected Target Coverage Algorithms for Industrial Wireless Sensor Networks," in IEEE Transactions on Industrial Informatics, vol. 13, no. 1, pp. 135-143, Feb. 2017.

[25] H. Feng and J. Dong, "Reliability analysis for WSN based on a modular k-out-of-n system," in Journal of Systems Engineering and Electronics, vol. 28, no. 2, pp. 407-412, April 2017. 
[26] A. S. Elsafrawey, E. S. Hassan and M. I. Dessouky, "Cooperative hybrid self-healing scheme for secure and data reliability in unattended wireless sensor networks," in IET Information Security, vol. 9, no. 4, pp. 223-233, 72015.

[27] G. Campobello, A. Leonardi and S. Palazzo, "Improving Energy Saving and Reliability in Wireless Sensor Networks Using a Simple CRT-Based Packet-Forwarding Solution," in IEEE/ACM Transactions on Networking, vol. 20, no. 1, pp. 191-205, Feb. 2012.

[28] M. Dong, K. Ota, A. Liu and M. Guo, "Joint Optimization of Lifetime and Transport Delay under Reliability Constraint Wireless Sensor Networks," in IEEE Transactions on Parallel and Distributed Systems, vol. 27, no. 1, pp. 225-236, Jan. 12016.

[29] X. Liu, N. Xiong, W. Li and Y. Xie, "An Optimization Scheme of Adaptive Dynamic Energy Consumption Based on Joint Network-Channel Coding in Wireless Sensor Networks," in IEEE Sensors Journal, vol. 15, no. 9, pp. 51585168, Sept. 2015.

[30] J. Long, M. Dong, K. Ota, A. Liu and S. Hai, "Reliability guaranteed efficient data gathering in wireless sensor networks," in IEEE Access, vol. 3, pp. 430-444, 2015

[31] J. M. Pak, C. K. Ahn, Y. S. Shmaliy and M. T. Lim, "Improving Reliability of Particle Filter-Based Localization in Wireless Sensor Networks via Hybrid Particle/FIR Filtering," in IEEE Transactions on Industrial Informatics, vol. 11, no. 5, pp. 1089-1098, Oct. 2015.

[32] B.S. Jayasri, G. Raghavendra Rao, "RaESS: Reliable-and-Efficient Statistical Spreading Data Fusion Mechanism in Wireless Sensor Network", Software Engineering Trends and Techniques in Intelligent Systems, CSoC, pp. 309-316, 2017

[33] Luo, H., Tao, H., Ma, H., \& Das, S. K. (2011). Data fusion with desired reliability in wireless sensor networks. IEEE Transactions on Parallel and Distributed Systems, 22(3), 501-513.

[34] Zhang, K., Li, C., \& Zhang, W. (2013). Wireless sensor data fusion algorithm based on the sensor scheduling and batch estimate. International Journal of Future Computer and Communication, 2(4), 333.

\section{BIOGRAPHIES OF AUTHORS}
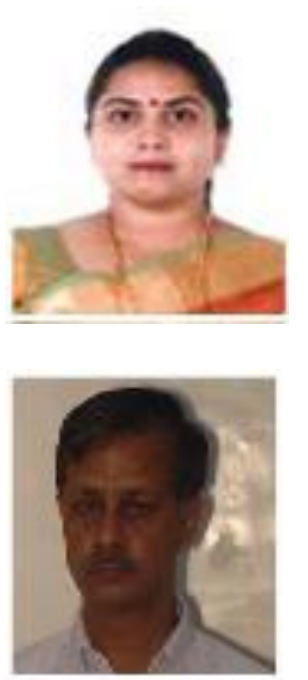

Jayasri B. S. is currently working as an Associate Professor in the department of CS\&E, The National Institutional of Engineering, and Mysuru. She obtained her M.tech degree in (2006) from Visvesvaraya Technical University, Belgaum. She is currently pursuing her Ph.D., and her area of interest includes Wireless Sensor Networks, Wireless Communication, Computer Networks, Operational Research and System Simulation Modeling.

G Raghavendra Rao took his BE ME and PhD degrees from UOM, IISC, Bangalore and UOM respectively. After serving as Asst. Engineer (Jaguar Project) at HAL, Bangalore for 4 years, he joined NIE as a faculty in June 1983. He was the first faculty of the department of Computer Science \& Engg., and was responsible for the establishment of the department, facilities and Laboratories of CS, IS and MCA departments in NIE. He became Asst. Professor in January 1990 and Professor of CSE in January 1995 respectively. He was Principal (I/C) of NIE from March 2004 to April 2008. He was also the founder Principal of NIE Institute of Technology from July 2008 to October 2010. 